\title{
Weighted Moving Average Method for Forecasting of Cryptocurrency Price: A Data Analytical Study on XRP Ripple Cryptocurrency
}

\author{
Nashirah Abu Bakar ${ }^{1}$ Sofian Rosbi ${ }^{2}$ and Kiyotaka Uzaki ${ }^{3}$ \\ ${ }^{1}$ Islamic Business School, College of Business, University of Utara Malaysia, Kedah, Malaysia \\ ${ }^{2}$ School of Mechatronic Engineering, University of Malaysia Perlis, Arau, Malaysia \\ ${ }^{3}$ Faculty of Economics, Oita University, Japan
}

\begin{abstract}
The aim of this study is to develop a reliable forecasting method for cryptocurrency namely XRP Ripple Cryptocurrency. The daily price of Ripple cryptocurrency collected from 1st October 2019 until 30th November 2019. This study implemented a forecasting method of simple moving average and the weighted moving average. The mean absolute percentage error for the simple moving average is $2.75 \%$. Meanwhile, the mean absolute percentage error for weighted moving average is $2.25 \%$. Therefore, the weighted moving average is more reliable forecasting method for predicting the price of Ripple cryptocurrency. The finding of this study helps investors to develop an investment portfolio with lower risk and higher returns.
\end{abstract}

Key Words: Forecasting, Moving Average, Ripple, Cryptocurrency.

\section{INTRODUCTION}

Cryptocurrency was started in 2008 by pioneer Satoshi Nakamoto. The most famous cryptocurrency is Bitcoin and first to be used widely. However, there are many cryptocurrencies exist, and generate high demand. The examples of cryptocurrencies are Litecoin, Ripple, Ethereum, Dogecoin and others. Many investors mention that invest in cryptocurrencies can generate high return, but the main point is either it's just a rumors or true. As suggested by Othman (2019) banks in GCC region are encouraged to either consider cryptocurrencies as an alternative investment asset for their portfolio investment diversification strategies or adopt the blockchain technology in their operation system to facilitate their customers with low transaction cost, high level of security and ease of use and real-time settlement. Therefore, statutory bodies of each country must take responsibility in monitoring agencies that involve in cryptocurrencies investment. This is because, cryptocurrencies are high risk investment. The value of Bitcoin cryptocurrency is increased sharply in 2017 that creates much attention from investors. According to Abu Bakar and Rosbi (2017) volatility of Bitcoin cryptocurrency is very high with the mean for Bitcoin return is 0.006 , deviation is 0.04458 and standard error is $4.458 \%$. Thus, investment in cryptocurrency must be monitor and need more attention from statutory body of each country.

As a result of the unprecedented pace of technological, money transfers and trades are implemented seriously on the internet in our contemporary world (Uyar and Kahraman, 2019). Thus, cryptocurrencies are getting high demand from investors. Cryptocurrency is a type of digital currency that using decentralized method for validating transaction. In the same time, the user of the Bitcoin is protected using hash algorithm. Cryptocurrency has no physical form of which no banknotes and coins exist, and which can only be transmitted via electronic means, typically allowing for instantaneous transactions and borderless transfer of ownership. It's also exists only in a network and in which encryption techniques are used to regulate the generation of units of currency. Bitcoin are difference with traditional money transaction. In fact, studies such as Abu Bakar, et al., (2017) have viewed the difference between cryptocurrency and traditional transaction. In definition, current fiat money is money in any form when in actual use or circulation as a medium of exchange, especially circulating banknotes and coins. This type of money is governmentissued currencies. Comparing to cryptocurrency, Bitcoin is digital currency in which encryption techniques are used to regulate the generation of units of currency. Cryptocurrency is a type of currency that is non-physical, of which no banknotes and coins exist. The transaction of Bitcoin only can be performed via electronic means, typically allowing for instantaneous transactions and borderless transfer of ownership. Therefore, Kostika and Laopodis (2019) suggested that the use of cryptocurrencies as a means of exchanging payment is the best example of technological innovations in the field of finance resulting in the removal of intermediaries such as banks. Therefore, the aim of this study is to develop reliable forecasting method for cryptocurrency namely XRP Ripple Cryptocurrency. XRP Ripple Cryptocurrency is fast, less costly and more scalable digital transaction. 


\section{LITERATURE REVIEW}

In the relevant current literature, studies on cryptocurrency are mostly concentrated on volatility of the price movement. From this point of view, it is widely used value at risk ( $\mathrm{VaR}$ ) method in measuring the Bitcoin cryptocurrency. Study by Stavroyiannis, (2018) found that Bitcoin cryptocurrency is highly volatile currency violating the value-at-risk measures more than the other assets. Uyar and Kahraman (2019) use value at risk method to compare investors of major conventional currencies and Bitcoin investors. The finding firstly, indicated that Bitcoin is found to be significantly risky with respect to the major currencies; and it is six times riskier than the singular riskiest currency. Second, in terms of inclusion of Bitcoin into a portfolio, which equally weights all currencies, it elevates overall portfolio risk by 98 per cent. While, Abu Bakar and Rosbi (2017) in presenting Bitcoin cryptocurrency, found that the high value of volatility for Bitcoin cryptocurrency indicates that the investment is categorize as high risk investment. Abu Bakar and Rosbi (2018) evaluate the data distribution of Ethereum exchange rate to validate the dynamic behavior of price movement. The finding indicates the distribution of first difference for Ethereum exchange rate is not a normal distribution data. Using Box-Whisker plot to detect the existence of outliers in the Ethereum data indicates there are suspected outliers and outliers in the Ethereum exchange rate data. The study concluded that Ethereum exchange rate data is highly volatile.

Study by Buchholz, et al., (2012) use ARCH/GARCH models to investigated the performance of Bitcoin. They found that before the peak of the bubble, volatility had a statistically significant positive effect on price. Then, after the bubble burst, they see that market participants feared holding Bitcoins because many realized that they could lose their wealth due to fluctuations in bitcoin price. Bariviera (2017) found the long memory content of daily volatility of Bitcoin is stronger than in daily returns Study by Cheong, (2019) regarding hedge of cryptocurrency indicated that cryptocurrencies can be a more effective hedge against FX risks as compared to other common hedging instruments and/or techniques such as gold or a diversified currency portfolio. Besides focus on the hedge of cryptocurrency, study by Kostika and Laopodis (2019) regarding short- and long-run dynamic linkages between selected cryptocurrencies found that cryptocurrencies do not interact with each other because the correlations of cryptocurrencies are weak and do not share a common long-run path; thus they are not cointegrated. The second results from the VAR method indicate the different reactions of each cryptocurrency to both exchange rate and equity shocks and that cryptocurrencies appear to be isolated from market-driven shocks. Third, the ups and downs in the cryptocurrencies' dynamic conditional correlations indicate that all cryptocurrencies were susceptible to speculative attacks and market events.

Inci and Lagasse (2019), investigates the role of cryptocurrencies in enhancing the performance of portfolios constructed from traditional asset classes. This study provided evidence that as a single investment, the best cryptocurrency is Ripple, followed by Bitcoin and Litecoin. Bitcoin is the best cryptocurrency enhancing the characteristics of the optimal portfolio. Ripple and Litecoin follow in terms of their usefulness in an optimal portfolio as single cryptocurrencies. Including all these cryptocurrencies in a portfolio generates the best (most optimal) results. While, study by Bejaoui, et al., (2019) regarding the dynamics of Bitcoin, Litecoin, Ethereum and Ripple daily returns and volatilities. The study suggested that market dynamics seem to be governed by two different states which differ from one cryptocurrency market to another. These findings can be explained by investors' behavior, i.e. speculative trading and herding behavior.

Balcilar, et al, (2017) investigate a non-parametric causality-in-quantiles test in order to analyze the causal relation between trading volume and Bitcoin returns and volatility reveals that the volume can predict returns over the quantile range of 0.25 to 0.75, i.e. except in Bitcoin bear and bull market regimes. Therefore, it is important to analyze the cryptocurrency transaction because this transaction is a new tool in digital currency. It also attracts more investors to invest in this transaction. Digital currency was allowed two willing parties to transact directly. This transaction is computationally impractical to reverse protect sellers from fraud, and routine escrow mechanisms could easily be implemented to protect buyers (Nakamoto, 2009). Cryptocurrency transaction is difference with traditional method of transaction. Cryptocurrency transaction must use blockchain network (Abu Bakar, et al., 2017; Abu Bakar and Rosbi, 2018).

Blockchains are a software protocol that underlie cryptocurrency in one sense, are nothing more than a modernizing information technology, but in another sense, are novel and disruptive (Yeoh, 2017). The blockchain is blocks of transaction history that shared publicly using secured cryptography. Each block contains the previous transaction information, timestamp and new transaction data in secured cryptographic hash programming language. Abu Bakar and Rosbi (2018) suggested that the security of blockchain is highly reliable because the implementation of hash algorithm. Hash algorithm is a mathematical algorithm that maps data of arbitrary size to a bit string of a fixed size (a hash) and is designed to be a one-way function, that is, a function which is infeasible to invert. However, the account holder of bitcoin including the receiver identity is anonymous exist in the system. Therefore, the account holder is undetected if any suspicious activities occurs in the transaction activity. Thus a new framework should be developing in order to meet the demand from the investors at the same can protect the investors from 
high risk in the cryptocurrency investment. This study contributes to the helps investors to develop investment portfolio with lower risk and higher return for XRP Ripple Cryptocurrency.

\section{METHODOLOGY}

This study evaluated two forecasting method to predict price of Ripple cryptocurrency. The two methods of forecasting are simple moving average (SMA) method and weighted moving average (WMA) method. Both of moving average method using three days of actual price for predicting next value of price for Ripple cryptocurrency.

The simple moving average is represented by Equation (1).

$P_{i}=\frac{P_{i-1}+P_{i-2}+P_{i-3}}{3}$

The parameters in Equation (1) are described as below:

$P_{i}$ : Predicted price of Ripple cryptocurrency on period $i$,

$P_{i-1}$ : Actual price of Ripple cryptocurrency on period $i-1$,

$P_{i-2}:$ Actual price of Ripple cryptocurrency on period $i-2$, and

$P_{i-3}:$ Actual price of Ripple cryptocurrency on period $i-3$.

The weighted moving average for this study represented as Equation (2).

$P_{i}=w_{a} P_{i-1}+w_{b} P_{i-2}+w_{c} P_{i-3}$

The parameters in Equation (2) are described as follow:

$w_{a}$ : The weighted of actual price of Ripple cryptocurrency on period $i$-1,

$w_{b}$ : The weighted of actual price of Ripple cryptocurrency on period $i-2$, and

$w_{c}$ : The weighted of actual price of Ripple cryptocurrency on period $i-3$.

In this study, the value of $w_{a}$ is $0.7, w_{b}$ is 0.2 and $w_{c}$ is 0.1 . The setting of this value makes prediction value sensitive to changes in previous observation of data to reduce error.

The error of forecasting is calculated using Equation (3).

Error $_{i}=P_{i}-A P_{i}$

The variables in Equation (3) are described as below:

Error $_{i}$ : Error on observation period $i$,

$P_{i}$ : Forecast value for price of Ripple cryptocurrency on observation period $i$, and

$A P_{i}$ : Actual value for price of Ripple cryptocurrency on observation period $i$.

The Shapiro-Wilk normality test performed using Equation (3).

$W=\frac{\left(\sum_{i=1}^{n} a_{i} x_{(i)}\right)^{2}}{\sum_{i=1}^{n}\left(x_{i}-\bar{x}\right)^{2}}$

The parameters in Equation (4) are explained as below:

$x_{(i)}$ : The $i$ th-order statistics with $x_{(1)}$ is lowest order.

$\bar{x}=\frac{x_{1}+x_{2}+x_{3}+\ldots+x_{n}}{n}:$ Sample mean for data of price for Ripple cryptocurrency.

The coefficient $a_{i}$ in Equation (4) is derived as Equation (5).

$\left(a_{1}, a_{2}, a_{3}, \ldots, a_{n}\right)=\frac{m^{\mathrm{T}} V^{-1}}{C}$

The variable $\mathrm{C}$ is a vector norm,

$C=\left\|V^{-1} m\right\|=\left(m^{\mathrm{T}} V^{-1} V^{-1} m\right)^{1 / 2}$ 
The variable $\mathrm{m}$, vector is described as below:

$m=\left(m_{1}, m_{2}, m_{3}, \ldots, m_{n}\right)^{\mathrm{T}}$

\section{RESULT AND DISCUSSIONS}

The objective of this study is to compare forecasting method of simple moving average and weighted moving average. The cryptocurrency price that selected for this study is XRP ripple cryptocurrency. The method in this study implemented three-days moving average calculation. Data collection for this study started from $1^{\text {st }}$ October 2019 until $30^{\text {th }}$ November 2019 . The number of daily observations is 60 days. The actual value of first observation ( $1^{\text {st }}$ October 2019) is USD 0.2550 for each unit of Ripple cryptocurrency. The actual value of $60^{\text {th }}$ observation $\left(30^{\text {th }}\right.$ November 2019) is USD 0.2294 for each unit of Ripple cryptocurrency. Figure 1 shows three lines of data represented by black line for real data, red line for simple moving average prediction method and blue line indicates dynamic behavior of weighted moving average for forecasting Ripple Cryptocurrency. Figure 1 concludes weighted moving is more appropriate forecasting method compared to simple moving average method. The weighted moving average predicted value of Ripple cryptocurrency close to real value rather than simple moving average.

Next, this study validating the error by checking the normality testing using histogram and Shapiro Wilk test. The purpose of the graphical and statistical testing of normality testing are to validate the forecasting method is reliable and trustworthy prediction method with comparison to real data.

Figure 2 shows histogram for data distribution of error for simple moving average method. Figure 2 shows the distribution of error is close to normal distribution line. Therefore, the distribution of error for simple moving average is consider distributed follow normal distribution. Table 1 shows result of statistical normality test using Shapiro-Wilk normality method for assessing normality for error of simple moving average compared to real data. Table 1 indicates the probability value ( $\mathrm{p}$-value) is 0.253 that is larger than alpha 0.05 . Therefore, error for simple moving average are normally distributed.

Figure 3 shows histogram for data distribution of error for weighted moving average method. Figure 3 shows the distribution of error is close to normal distribution line. Therefore, the distribution of error for weighted moving average is consider distributed follow normal distribution. Table 2 shows result of statistical normality test using Shapiro-Wilk normality method for assessing normality for error of weighted moving average compared to real data. Table 2 indicates the probability value ( $\mathrm{p}$-value) is 0.058 that is larger than alpha 0.05 . Therefore, error for weighted moving average are normally distributed.

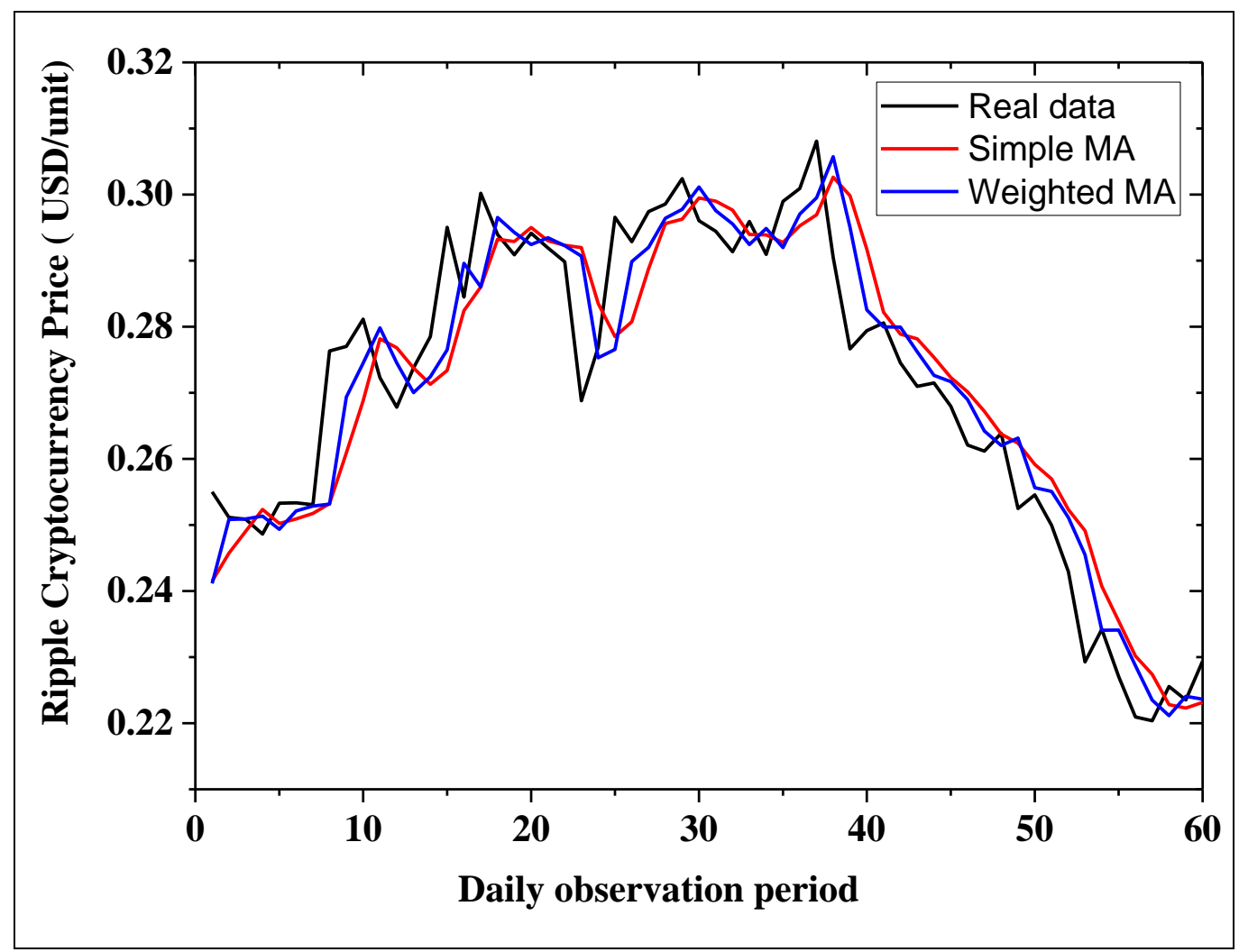

Figure 1: The behavior of cryptocurrency price with prediction 


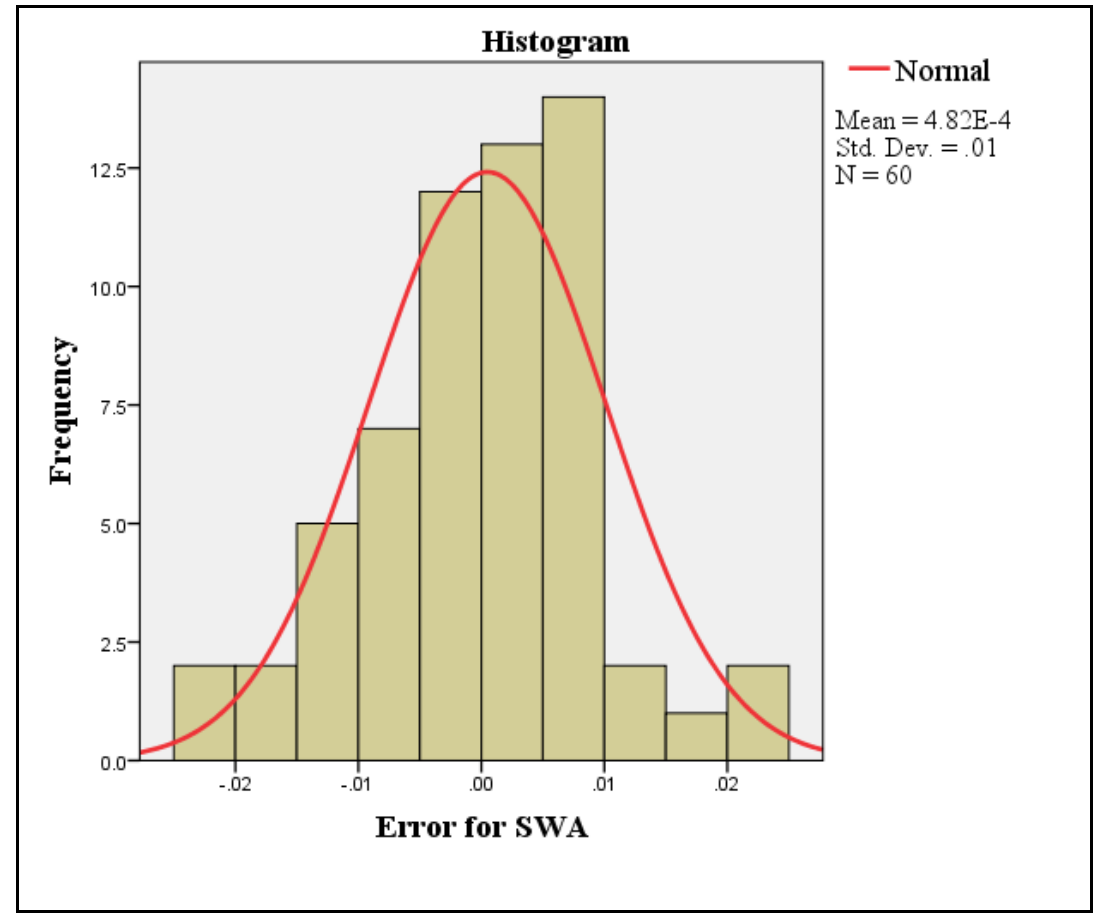

Figure 2: Normality checking for error of simple moving average

Table 1: Statistical normality test for distribution of error for simple moving average

\begin{tabular}{|c|c|c|c|}
\hline \multicolumn{4}{|c|}{ Shapiro-Wilk normality test } \\
\hline Statistics & Degree of freedom & Significant of Probability value & Comment \\
\hline 0.975 & 60 & 0.253 & Normally distributed \\
\hline
\end{tabular}

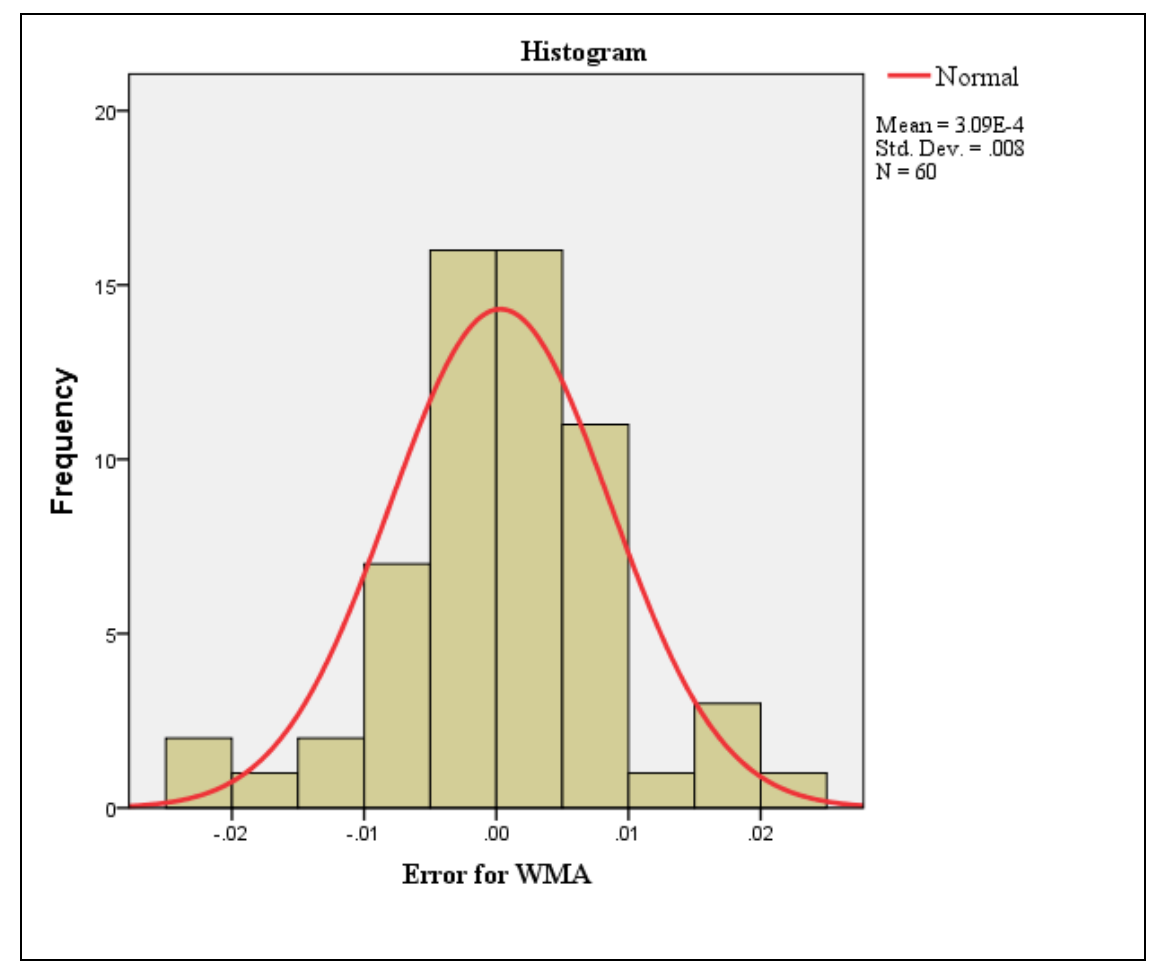

Figure 3: Normality checking for error of simple moving average

Table 2: Statistical normality test for distribution of error for weighted moving average

\begin{tabular}{|c|c|c|c|}
\hline \multicolumn{4}{|c|}{ Shapiro-Wilk normality test } \\
\hline Statistics & Degree of freedom & Significant of Probability value & Comment \\
\hline 0.962 & 60 & 0.058 & Normally distributed \\
\hline
\end{tabular}


Next, this study checked absolute percentage error for both of prediction methods namely simple moving average and weighted moving average. Figure 4 shows comparison of absolute percentage error between simple moving average and weighted moving average. The comparison between these two prediction methods shows weighted moving average contributed less absolute percentage error than simple moving average. Therefore, weightage moving average is more suitable and reliable for forecasting Ripple cryptocurrency price movement. This finding is confirmed by calculating mean absolute percentage error as shown in Table 3. Mean absolute percentage error for simple moving average is $2.75 \%$. Meanwhile, mean absolute percentage error is $2.25 \%$. This study concluded the forecasting of Ripple cryptocurrency price movement using weighted moving average is reliable with less error.

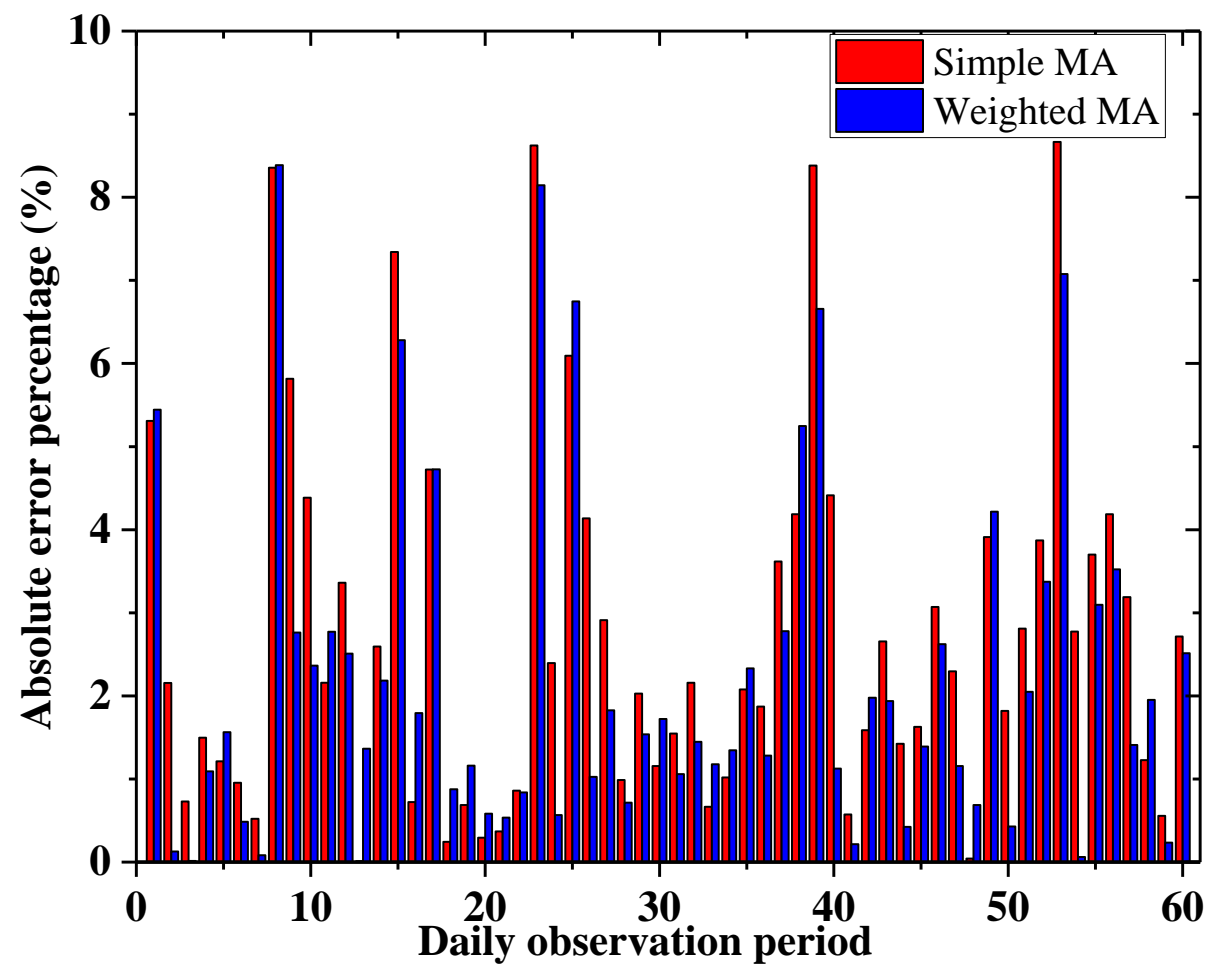

Figure 4: Absolute percentage error analysis for simple moving average and weightage moving average

Table 3: Mean absolute percentage error for two prediction methods

\begin{tabular}{|l|c|}
\hline \multicolumn{1}{|c|}{ Method } & Mean absolute error percentage (MAPE) \\
\hline Simple moving average (SMA) & $2.75 \%$ \\
\hline Weightage Moving Average (WMA) & $2.25 \%$ \\
\hline
\end{tabular}

\section{CONCLUSION}

The aim of this study is to develop forecasting method using simple moving average and weighted moving average method for Ripple cryptocurrency. The main findings of this study are:

a. Data collection for this study started from $1^{\text {st }}$ October 2019 until $30^{\text {th }}$ November 2019 . The number of daily observations is 60 days. The actual value of first observation $\left(1^{\text {st }}\right.$ October 2019$)$ is USD 0.2550 for each unit of Ripple cryptocurrency. The actual value of $60^{\text {th }}$ observation $\left(30^{\text {th }}\right.$ November 2019) is USD 0.2294 for each unit of Ripple cryptocurrency.

b. In developing reliable forecasting method for two methods of moving average, the distribution or error is evaluated with statistical test and graphical test of normal distribution. Result show error from simple moving average and weightage moving average are follow normal distribution.

c. This study calculated mean absolute percentage error to validate the reliability of forecasting method. Mean absolute percentage error for simple moving average is $2.75 \%$. Meanwhile, mean absolute percentage error is $2.25 \%$. This study 
concluded the forecasting of Ripple cryptocurrency price movement using weighted moving average is reliable with less error.

\section{ACKNOWLEDGMENT}

The authors would like to thank Institute for Management and Business Research (IMBRe) of Universiti Utara Malaysia (UUM) for their financial support under research grant scheme "Case Study Research Grant" SO code number 14501.

\section{REFERENCES}

1. Abu Bakar, N. and Rosbi, S. (2017). High Volatility Detection Method Using Statistical Process Control for Cryptocurrency Exchange Rate: A Case Study of Bitcoin. The International Journal of Engineering and Science, 6 (11), $39-48$.

2. Abu Bakar, N. and Rosbi, S. (2017). Identification of Non-Equilibrium Growth for Bitcoin Exchange rate: Mathematical Derivation Method in Islamic Financial Engineering. International Journal of Scientific Research and Management, 5 (12), 7772-7781.

3. Abu Bakar, N. and Rosbi, S. (2017). Robust Statistical Pearson Correlation Diagnostics for Bitcoin Exchange Rate with Trading Volume: An Analysis of High Frequency Data in High Volatility Environment. International Journal of Advanced Engineering, Management and Science, 3 (12), 1135-1142.

4. Abu Bakar, N. and Rosbi, S. (2018). Pearson Product Moment Correlation Diagnostics Between two types of cryptocurrencies: A case study of Bitcoin and Ethereum. International Journal of Advances in Scientific Research and Engineering, 4 (12), 40-51.

5. Abu Bakar, N. and Rosbi, S. (2018). Robust Framework Diagnostics of Blockchain for Bitcoin Transaction System: A Technical Analysis from Islamic Financial Technology (i-FinTech) Perspective. International Journal of Business and Management, 2 (3), 22-29.

6. Abu Bakar, N. and Rosbi, S. (2018). Robust Outliers Detection Method for Ethereum Exchange Rate: A Statistical Approach Using High Frequency Data. The International Journal of Engineering and Science, 7 (4), 01-08.

7. Abu Bakar, N., Rosbi, S. and Uzaki, K. (2017). Cryptocurrency Framework Diagnostics from Islamic Finance Perspective: A New Insight of Bitcoin System Transaction. International Journal of Management Science and Business Administration, 4 (1), 19-28.

8. Balcilar, M., Bouri, E., Gupta, R., and Roubaud, D. (2017). Can Volume Predict Bitcoin Returns and Volatility? A Quantiles-Based Approach, Economic Modelling, 64, 74 - 81.

9. Bariviera, A.F. (2017). The Inefficiency of Bitcoin Revisited: A Dynamic Approach, available at: https://arxiv.org/pdf/1709.08090.pdf (accessed 10 April 2018)

10. Bejaoui, A., Ben Sassi, S. and Majdoub, J. (2019). Market dynamics, cyclical patterns and market states: Is there a difference between digital currencies markets?. Studies in Economics and Finance.

11. Buchholz, M., Delaney, J., Warren, J. and Parker, J. (2012). Bits and Bets, Information, Price Volatility, and Demand for BitCoin, Economics 312, available at: http:// www.bitcointrading.com/pdf/bitsandbets.pdf

12. Cheong, C. (2019). Cryptocurrencies vs global foreign exchange risk. Journal of Risk Finance, 20 (4), $330-351$.

13. Inci, A. and Lagasse, R. (2019). Cryptocurrencies: applications and investment opportunities. Journal of Capital Markets Studies, 3 (2), 98-112.

14. Kostika, E. and Laopodis, N. (2019). Dynamic linkages among cryptocurrencies, exchange rates and global equity markets. Studies in Economics and Finance.

15. Nakamoto, S., (2009). Bitcoin: A Peer-to-Peer Electronic Cash System, available at: http://bitcoin.org/bitcoin.pdf (accessed 20 October 2017)

16. Othman, A., Alhabshi, S., Kassim, S. and Sharofiddin, A. (2019). The impact of cryptocurrencies market development on banks' deposits variability in the GCC region. Journal of Financial Economic Policy.

17. Stavroyiannis, S. (2018). Value-at-risk and related measures for the Bitcoin. Journal of Risk Finance, 19 (2), $127-136$.

18. Uyar, U. and Kahraman, I. (2019). The risk analysis of Bitcoin and major currencies: value at risk approach. Journal of Money Laundering Control, 22 (1), 38-52.

19. Yeoh, P. (2017). Regulatory issues in blockchain technology. Journal of Financial Regulation and Compliance, 25(2), 196-208. 